\title{
Full Physical Optics Sky Coverage Simulation for MCAO Systems on ELT's
}

\author{
Lianqi Wang $^{1, a}$, Brent Ellerbroek ${ }^{1}$, and Jean Pierre Veran ${ }^{2}$ \\ 1 TMT Observatory Corporation, 2632 E Washington Blvd, Pasadena, CA, 91107, USA \\ 2 Herzberg Institute of Astrophysics, 5071 West Saanich Road Victoria, BC V9E 2E7 Canada
}

\begin{abstract}
For multi-conjugate and multi-object AO (MCAO and MOAO) systems, the evaluation of sky coverage is challenging because asterisms of three or more natural guide stars (NGS's) must be considered. In this paper, we described a full rank, physical optics sky coverage simulation tool for modeling both the NGS and LGS AO control loops of MCAO systems on ELT's. Sufficient computational efficiency to enable practical Monte Carlo simulations over a large number of natural guide star asterisms can be obtained using the so-called "split tomography" control architecture, in which the higher-order wavefront correction computed from the LGS WFS measurements is not affected by the lower-order NGS control loop. We first compute and store time histories of 1) the atmospheric modes that are blind to LGS WFS and 2) natural guide star point spread functions for a full ensemble of many NGS over the course of a single high order AO simulation run. The behavior of the NGS loop may then be evaluated separately for each NGS asterism in a post-processing step to derive sky coverage statistics. This post-processing analysis also helps us find the best approach to WFS centroiding to maximize skycoverage.
\end{abstract}

\section{Introduction}

For a multi-conjugate or multi-object adaptive optics system with a non-negligible field of view (FoV), multiple NGS WFSs are needed to provide measurements of both global tip/tilt and tilt anisoplanatism (i.e., field varying tip/tilt). The "sky coverage" of an AO system is conventionally expressed as the cumulative probability of obtaining a given level of wavefront correction for an arbitrarily selected science object. High fidelity simulations of MCAO systems in time domain are time consuming because rigorous sky coverage estimates must be based upon simulations performed with a large number of randomly generated NGS asterisms. Previous sky coverage analysis of LGS MCAO and MOAO systems $[1,2]$ have therefore employed much simplified simulation tools based upon 1) linear, Zernikebased models of wavefront sensing and correction on an ideal circular pupil, 2) an idealized linear model of the high-order LGS wavefront control loop, and 3) a simplified, time-averaged model for the degree of NGS "sharpening" provided by the higher-order LGS adaptive optics as a function of the NGS location in the AO system FoV. The above approach provides a detailed analysis of some, but not all, of the potential tip/tilt error sources which must be considered when evaluating sky coverage. In particular, the residual errors due to tilt anisoplanatism, servo lag, and telescope wind shake can be calculated using these tools. But evaluating the tip/tilt errors due to other important effects including NGS WFS measurement noise, time-varying aberrations of the partially compensated NGS PSF, and under-sampling of the NGS PSF by the NGS WFS detector requires more rigorous simulation models. These include physical optics modeling of the NGS WFS, and a simulation of the higher-order LGS control loop.

In this paper, we describe a high fidelity, time domain simulation for a complete NGS/LGS MCAO control architecture, which can be used to address all these limitations of the previous sky coverage analysis. Section 2 reviews the hardware and control system parameters for the TMT Narrow Field Infrared Adaptive Optics System (NFIRAOS) and the basic theory to do the sky coverage. Section 3 summaries the system and atmospheric parameters and several control algorithm refinements and parameter trade studies found to be useful in improving sky coverage. Section 4 outlines the sky coverage results for NFIRAOS.

a e-mail: lianqiw@tmt.org

This is an Open Access article distributed under the terms of the Creative Commons Attribution-Noncommercial License, which permits unrestricted use, distribution, and reproduction in any noncommercial medium, provided the original work is properly cited. 


\section{The NFIRAOS LGS MCAO System and "Split Tomography" Control}

Our analysis is based on the TMT NFIRAOS [3], which is an order 60x60 dual conjugate AO system with two deformable mirrors (DMs) conjugated to range of 0.0 and $11.3 \mathrm{~km}$ respectively, an asterism of 6 sodium laser guide stars arranged in a pentagon with a 35" radius plus one more on-axis, and up to three natural guide star low order wavefront sensors (1 tip/tilt/focus/astigmatism (TTF) and up to 2 tip/tilt only). The ground-conjugate DM is mounted on a tip/tilt stage (TTS) that has limited bandwidth (we measured $90 \mathrm{~Hz}$ on the TTS prototype made by CILAS). A woofer/tweeter type II control law is implemented that uses the TTS to compensate the low temporal frequency, high stroke tip/tilt wavefront errors, and uses the DM to control the high temporal frequency, but low stroke tip/tilt errors [4].

The performance requirements for NFIRAOS include diffraction-limited turbulence compensation over fields of view of up to 30 " in diameter (a square FoV of size 10 " $\times 10$ " is used for performance evaluation in this paper). The patrol field for the low-order NGS WFS is a larger, non-vignetted, 2' diameter circular FoV. The NGS are acquired via probe arms which patrol outside of the science FoV. The NGS WFS will operate in the near infrared ( $\mathrm{J}$ and $\mathrm{H}$ bands) since 1) the NGS images will be partially "sharpened" by the NFIRAOS system, and 2) more guide stars are available in the near infrared.

The split tomography control algorithms [5] as adopted by NFIRAOS will be used in these sky coverage coverage simulations. In split tomography, the separate LGS and NGS control loops are driven independently. The atmospheric tomography step of the LGS control loop applies a minimum variance estimator to tip/tilt removed, pseudo open loop LGS WFS gradients. The NGS control loop uses a noise-weighted, least square reconstructor, and usually operates at a different (slower) frame rate than the LGS loop depending upon the brightness and location in the FoV of the NGS.

The NGS controlled modes are the tip/tilt and (largely) tilt anisoplanatism modes that produce pure tip/tilt in each LGS wavefront, and are therefore not sensed by the tilt-removed LGS WFS. Most of the error in the tilt anisoplanatism modes can be corrected by applying a combination of three quadratic Zernike modes with proportional amplitudes in two conjugate planes[6]. For a two DM MCAO system like the TMT NFIRAOS, the following equations describe the global tip/tilt modes $\left(\mathbf{m}_{1}\right.$ and $\left.\mathbf{m}_{2}\right)$ and these three dominant tilt anisoplanatism modes $\left(\mathbf{m}_{3}\right.$ to $\left.\mathbf{m}_{5}\right)$ that must be measured using the low order NGS WFS:

$$
\begin{aligned}
& \mathbf{m}_{1}=\left(\mathbf{x}_{\mathbf{0}} ; \mathbf{0}\right), \quad \mathbf{m}_{2}=\left(\mathbf{y}_{\mathbf{0}} ; \mathbf{0}\right), \quad \mathbf{m}_{3}=\left(\left[\mathbf{x}_{\mathbf{0}}^{2}+\mathbf{y}_{\mathbf{0}}^{2}\right] ;-\left[\mathbf{x}_{\mathbf{1}}^{2}+\mathbf{y}_{\mathbf{1}}^{2}\right] / s_{c}^{2}\right), \\
& \mathbf{m}_{4}=\left(\left[\mathbf{x}_{\mathbf{0}}^{\mathbf{2}}-\mathbf{y}_{\mathbf{0}}^{\mathbf{2}}\right] ;-\left[\mathbf{x}_{\mathbf{1}}^{\mathbf{2}}-\mathbf{y}_{\mathbf{1}}^{\mathbf{2}}\right] / s_{c}^{2}\right), \quad \mathbf{m}_{5}=\left(\left[\mathbf{x}_{\mathbf{0}} \mathbf{y}_{\mathbf{0}}\right] ;-\left[\mathbf{x}_{\mathbf{1}} \mathbf{y}_{\mathbf{1}}\right] / s_{c}^{2}\right), \quad M=\left(\mathbf{m}_{1}\left|\mathbf{m}_{2}\right| \mathbf{m}_{3}\left|\mathbf{m}_{4}\right| \mathbf{m}_{5}\right),
\end{aligned}
$$

where $\mathbf{x}_{\mathbf{0}}, \mathbf{y}_{\mathbf{0}}$ and $\mathbf{x}_{\mathbf{1}}, \mathbf{y}_{\mathbf{1}}$ are the actuator coordinates on the ground and upper DM. The term $s_{c}=$ $1-h_{c} / h_{s}$ accounts for the cone effect for a DM conjugated to range $h_{c}$ and an LGS at range $h_{s}(90 \mathrm{~km}$ in our simulations). The global tip/tilt mode vectors $\mathbf{m}_{1}$ and $\mathbf{m}_{2}$ have the tip/tilt Zernike modes applied to the ground DM only. The tilt anisoplanatism mode vectors $\mathbf{m}_{3}$ to $\mathbf{m}_{5}$ each contains a quadratic Zernike mode with proportional amplitudes on the ground and upper DMs, whose combined effect produces pure tip/tilt in the LGS wavefront sensors, but contains field dependent tip/tilt (plate scale effect) and quadratic wavefront aberrations across the science field and NGS patrol field. These tilt anisoplanatism errors are therefore named plate scale modes. It is worth pointing out that these plate scale modes arise solely from the fact that the LGS is propagated from a finite altitude while the NGS and science are from infinity.

Detailed formulation of the split tomography approach can be found in [5]. We summarize the measurement and control of the NGS modes based upon the NGS WFS measurements here:

$$
a=P_{L} a_{L}+a_{N}, \quad s_{N}=G_{N}\left[H_{x}^{N} x-H_{a}^{N} a\right], \quad \delta a_{N}=M G_{M}^{\dagger} s_{N} .
$$

Here $a_{L}$ and $a_{N}$ are the DM actuator commands computed from the LGS tomography and NGS measurements, $a$ is the total DM command, $P_{L}$ is the NGS mode removal operator for the LGS DM commands, $x$ is the atmospheric turbulence optical path difference (OPD) defined on planes conjugated to several (six in our case) different altitudes, $H_{x}^{N}$ and $H_{a}^{N}$ are interpolation operators for the influence of the turbulence OPD and DM actuators on the rays (in $1 / 64 \mathrm{~m}$ sampling) traced from the NGS to 
the aperture plane, $G_{N}$ is the NGS WFS gradient operator which computes the average gradient of the turbulence OPD (in geometrical simulations) or the center of gravity for the images formed (in physical optics simulations) in each of the subapertures, the NGS mode matrix $M$ represents the NGS modes in terms of DM actuator commands, $G_{M}=G_{N} H_{a}^{N} M$, a five-column matrix, is the influence matrix from the 5 NGS modes onto NGS WFS measurements, and finally $\delta a_{N}$ is the estimate of the residual closed-loop error in the NGS modes that is added into $a_{N}$ after servo filtering. The NGS modal reconstructor $G_{M}^{\dagger}$ implements a least square estimation weighted by the NGS measurement noise, and is computed using

$$
\hat{a}=\arg \min \left\|G_{M} a-s^{N}\right\|_{C_{N}^{-1}}=G_{M}^{\dagger} s^{N}, \quad G_{M}^{\dagger}=\left(G_{M}^{T} C_{N}^{-1} G_{M}\right)^{-1} G_{M}^{T} C_{N}^{-1},
$$

where $C_{N}^{-1}$ is the inverse of the NGS measurement error covariance matrix.

The projection matrix $P_{L}$ is used to minimize the cross-coupling between the LGS and NGS measurements. In the original split-tomography approach, $P_{L}$ was chosen so that the NGS WFS gradient generated by $P_{L} a_{L}$ will not be reconstructed into any NGS mode, which gives $P_{L}=I-M G_{M}^{\dagger} G_{N} H_{a}^{N}$. Notice that $G_{M}^{\dagger}$ and $C_{N}^{-1}$, and consequently $P_{L}$, then depend upon the choice of NGS asterism.

This split tomography control architecture enables the efficient modeling and evaluation of the LGS and NGS control loops as a two step process. The first step is a simulation of the high-order LGS control loop using our Linear Adaptive Optics Simulator (LAOS) code [7]. During this step, the five NGS-controlled modes contained in the atmosphere are corrected perfectly, without the degrading effects of WFS noise or servo delay, to minimize the wavefront error over the science FoV. A time history of the resulting NGS WFS point spread functions (PSFs), the best fit of NGS modes, and the associated geometric gradient measurements, are recorded for an array of both TT and TTF wavefront sensors (WFS) positioned at 29 NGS locations arranged on a grid with a spacing of 20" across the 2' patrol FoV.

During the post-processing step, for each random NGS asterism, we will replay the movie of the higher order loop and estimate the actual correction to the NGS modes from the NGS measurements, which is computed from the saved NGS WFS PSFs (in physical optics simulations) or geometric gradient measurements (in geometric simulations), and the best-fit NGS modes.

Accurate sky coverage estimates require the simulation of hundreds of NGS asterisms, and we must break the dependence between the LGS and NGS WFS measurements in Equation 2 so that we can estimate the performance of the NGS loop without rerunning the full simulation of LGS tomography. We change the order of computation by first applying an ideal correction of the NGS modes during the higher order LGS simulation,

$$
\begin{aligned}
m^{*} & =\left(H_{a}^{s c} M\right)^{\dagger}\left(H_{x}^{s c} x-H_{a}^{s c} P_{L}^{\prime} a_{L}\right), \quad,\left(H_{a}^{s c} M\right)^{\dagger}=\left(M^{T} H_{a}^{s c T} W_{a} H_{a}^{s c} M\right)^{-1} M^{T} H_{a}^{s c T} W_{a}, \\
a^{*} & =P_{L}^{\prime} a_{L}+M m^{*}, \quad s_{N}^{*}=G_{N}\left[H_{x}^{N} x-H_{a}^{N} a^{*}\right],
\end{aligned}
$$

where $H_{x}^{s c}$ and $H_{a}^{s c}$ are interpolation operators for the influence of the turbulence OPD and DM actuators on the rays traced from the science objects to the aperture plane. $\left(H_{a}^{s c} M\right)^{\dagger}$ is the least square reconstructor to compute the best fit of the NGS modes onto the residual OPDs in the science field, for the diagonal weighting matrix $W_{a}$ composed of the aperture amplitude function. The optimal fit NGS mode correction $m^{*}$ is then added to the NGS mode removed LGS DM actuator command without servo filtering to obtain the total DM command $a^{*}$, which is applied during the higher order closed loop simulation. The NGS measurement $s_{N}^{*}$ with ideal NGS mode correction applied is saved for geometric simulations during the post-processing step.

We have changed $P_{L}$ in Equation 2 to $P_{L}^{\prime}$ in Equation 4 for efficient post-processing, since $P_{L}$ cannot be constructed simultaneously for multiple asterisms with individual values for $G_{M}$ and $C_{N}^{-1}$. We choose to orthogonalize the influence of the LGS and NGS mode wavefront errors in the science field by imposing $\left(H_{a}^{s c} M\right)^{\dagger} H_{a}^{s c} P_{L}^{\prime} a_{L}=0$, which gives $P_{L}^{\prime}=I-M\left(H_{a}^{s c} M\right)^{\dagger} H_{a}^{s c}$. Simulations for sample asterisms show that this modification degrades NGS loop performance only marginally.

The NGS control loop performance can now be simulated and evaluated in post-processing for an arbitrary asterism. Let $m$ be the actual NGS modes (a 5-element vector) applied at certain time step for 
First conference on Adaptive Optics for Extremely Large Telescopes

Table 1. The six-layer turbulence profile typical of Cerro Pachon. The height $h_{i}$, relative turbulence strength $\gamma_{i}$, and wind speed $v_{i}$ are shown for each layer $i$.

\begin{tabular}{crrr}
\hline Layer $i$ & $h_{i}(\mathrm{~m})$ & $\gamma_{i}$ & $v_{i}(\mathrm{~m} / \mathrm{s})$ \\
\hline 1 & 0 & 0.6523 & 5 \\
2 & 2577 & 0.1723 & 13 \\
3 & 5155 & 0.0551 & 20 \\
4 & 7732 & 0.0248 & 30 \\
5 & 12887 & 0.0736 & 20 \\
6 & 15464 & 0.0219 & 10 \\
\hline
\end{tabular}

a given asterism, the closed loop correction $\delta m$ is then

$$
\delta m=G_{M}^{\dagger} \operatorname{CoG}\left(\mathrm{I}\left(\theta ; m^{*}-m\right)+n\right),
$$

in physical optics simulations, where we use Fourier optics techniques to compute NGS PSFs, sample them onto detector pixels to obtain $\mathrm{I}\left(\theta, m^{*}-m\right)$ (where $\theta$ denotes NGS direction), and finally apply a centroiding method to obtain the NGS WFS measurement. The term $n$ denotes various error sources (e.g. photon and detector read noise). The closed loop correction $\delta m$ is then added to $m$ after servo filtering as described.

\section{Sky Coverage Simulation Parameters, Methods, and Trade Studies}

The nominal sky coverage simulations are carried out for median seeing conditions (Fried parameter $r_{0}=0.15 \mathrm{~m}$, outer scale $L_{0}=30 \mathrm{~m}$ ) at the Galactic Pole. The 6-layer turbulence profile typical of Cerro Pachon [8], as shown in Table 1, is used for the simulation. The telescope wind shake of 20 mas under 75 percentile condition is added to the input tip/tilt disturbance to simulate the wind shake effect. The $1 k \times 1 k$ Teledyne Hawaii-1RG near infrared detector with $5 e^{-}$read out noise, and 0 dark current is chosen as the baseline. The end-to-end optical throughput of the whole system is 0.4 . The detector passband is chosen as $\mathrm{J}$ plus $\mathrm{H}$ with central frequencies of 1.25 and $1.65 \mu \mathrm{m}$ respectively. The detector quantum efficiency is 0.8 in both $\mathrm{J}$ and $\mathrm{H}$ bands. The Detector pixel count is $1024 \times 1024$ during acquisition and $4 \times 4$ in closed loop run. The sky background is 16.25 and 14.40 magnitude/ $\operatorname{arcsec}^{2}$ in $\mathrm{J}$ and $\mathrm{H}$ bands respectively. The intensity of zero magnitude star is 3.77 and $3.17 \times 10^{9}$ photons $/ \mathrm{m}^{2} / \mathrm{s}$ in $\mathrm{J}$ and $\mathrm{H}$ bands [9]. The NGS limiting magnitude is 22.

During the sky coverage simulation, 500 different random star fields are generated from the Besancon star count model[10,11]. For each star field the post-processing evaluates the optimal NGS sampling frequency and obtain the minimum wavefront error in addition to the error due to noise and turbulence. The sky coverage cumulative distribution function is then obtained by sorting the results for the 500 star fields.

Several simulation upgrades and trade studies have been performed to obtain accurate and optimal results using this basic simulation approach. These include:

1. For physical optics simulations, the impact of the residual NGS modes $m^{*}-m$ upon the NGS PSF and the NGS WFS measurement $s_{N}$ is implemented by applying both the tip/tilt and quadratic components of the residual NGS modes and then recomputing the PSF, instead of simply shifting the PSF by the tip/tilt component. This is necessary for accurate results, since the impact of the quadratic wavefront aberrations is non-trivial.

2. Type II control of the plate scale modes, and improved type II control of tip/tilt (to account for the measured $90 \mathrm{~Hz}$ response of the actual TTS hardware) have been implemented to reduce the error due to servo lag.

3. Adaptively "demoting" the TTF WFS by averaging the gradient measurements from the four subaperture to simulate a TT WFS for those asterisms where this improves performance.

4. Adjusting the WFS pixel size to $\lambda_{H} / D$ to fully contain the core of the diffraction limited PSF in $2 \times 2$ pixels to improve the SNR, since detector read noise is non-negligible.

5. For physical optics simulations, the classical matched filter [12] for estimating the motion of the NGS images outperforms the constrained matched filter previously developed for LGS WFS applications[13], primarily on account of the amplified sensitivity to noise of the constrained matched filter when used with sub-pixel images. 


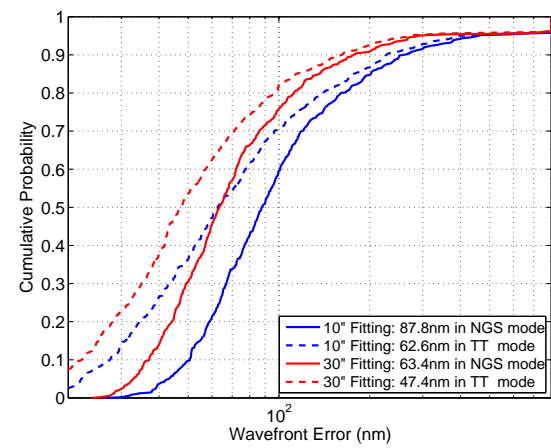

Figure 1. Sky coverage results for nominal system and atmospheric conditions.

6. Reoptimizing the high order DM fitting optimization to a larger field of view (30" circular FoV) helps to gain much better sharping in the NGS directions without impacting the performance in the science directions significantly.

\section{Sky Coverage Results}

We have validated the sky coverage post-processing simulation against integrated LAOS simulations of both LGS and NGS mode control using 4 symmetrical and asymmetrical asterisms with a range of NGS wavefront sensing options. The post-processing method agrees very well with the integrated simulation.

Figure 1 shows the wavefront errors as a function of sky coverage in the tip/tilt and the full set of NGS modes for nominal system and atmospheric conditions. Results for two DM optimization schemes are shown, DM optimized for the 10 " $\times 10$ " science field, and DM optimized for a larger 30" circular FoV. The wavefront error in overall NGS modes are reduced from $87.8 \mathrm{~nm}$ to $63.4 \mathrm{~nm}$ by simply reoptimizing the DM fitting FoV. The impact on science objects are small relative to this gain.

\section{Acknowledgment}

This work is supported by the National Science Foundation Science and Technology Center for Adaptive Optics, managed by the University of California at Santa Cruz under cooperative agreement No. AST-9876783.

This work is also supported by the Thirty Meter Telescope (TMT) project. The authors gratefully acknowledge the support of the TMT partner institutions. They are the Association of Canadian Universities for Research in Astronomy (ACURA), the California Institute of Technology and the University of California. This work was supported as well by the Gordon and Betty Moore Foundation, the Canada Foundation for Innovation, the Ontario Ministry of Research and Innovation, the National Research Council of Canada, the Natural Sciences and Engineering Research Council of Canada, the British Columbia Knowledge Development Fund, the Association of Universities for Research in Astronomy (AURA) and the U.S. National Science Foundation.

\section{References}

1. R.M. Clare, B.L. Ellerbroek, G. Herriot, J.P. Véran, Appl. Opt. 45, 8964 (2006)

2. R.M. Clare, B.L. Ellerbroek, Journal of the Optical Society of America A 23, 418 (2006)

3. B. Ellerbroek, S. Adkins, D. Andersen et al.,Progress toward developing the TMT adaptive optical systems and their components, SPIE Conference Series (2008), Vol. 7015

4. J.P. Veran, G. Herriot, Type II Woofer-Tweeter Control for NFIRAOS on TMT, in OSA Technical Digest (CD) (2009)

5. L. Gilles, B.L. Ellerbroek, Journal of the Optical Society of America A 25, 2427 (2008)

6. B.L. Ellerbroek, F. Rigaut, Journal of the Optical Society of America A 18, 2539 (2001)

7. L. Gilles, B. Ellerbroek, J.P. Véran, LGS MCAO performance of the Thirty Meter Telescope with elongated beacons and matched filtering, in SPIE Conference Series (2006), Vol. 6272

8. J. Vernin, A. Agabi, R. Avila, et al., Gemini Document RTP-AO-G0094(2000)

9. M.S. Bessell, J.M. Brett, Pub. Astron. Soc. Pacific 100, 1134 (1988)

10. A.C. Robin, C. Reylé, S. Derrière, S. Picaud, Astronomy and Astrophysics 409, 523 (2003)

11. http://model.obs-besancon.fr/

12. L. Gilles, B. Ellerbroek, Appl. Opt. 45(25), 6568 (2006),

13. L. Gilles, B.L. Ellerbroek, Optics Letters 33, 1159 (2008) 